\title{
Application of distributed optical fiber sensors to borehole temperature and strain monitoring
}

\author{
Dr.-Ing. Nils Nöther \\ fibrisTerre GmbH / BAM Bundesanstalt für Materialforschung und Prüfung \\ Köpenicker Str. 154, 10997 Berlin \\ [Nils Nöther, Marko Krcmar, Stefan von der Mark (fibrisTerre GmbH / BAM Bundesanstalt für \\ Materialforschung und -prüfung), Jan Henninges, Thomas Reinsch (Helmholtz Centre \\ Potsdam, GFZ German Research Centre for Geosciences)]
}

\section{INTRODUCTION}

For long-range measurements in geotechnical and industrial applications, distributed optical fiber sensors have become a tool of increasing importance throughout the past decade. Classic deformation monitoring (performed by strain gauges etc.) and temperature monitoring (Pt100 and alike) deliver data from fixed, single spots of a structure; quasi-distributed measurements (fiber bragg gratings) provide a chain of discrete measurement points along a limited sensing length. In contrast, an optical fiber connected to a device for distributed strain and temperature sensing (DTSS) will provide a continuous profile of strain and temperature - spatially resolved down to less than $1 \mathrm{~m}$ - over a range of several tens of kilometers.

In DTSS measurements, the nonlinear optical effect of stimulated Brillouin scattering (SBS) is employed: two light waves with a stable, tunable frequency offset are injected into opposite ends of the sensing fiber, where they will form a beat pattern, at which parts of the light will be scattered. By matching the frequency offset of the light waves to the propagation of acoustic fluctuation in the optical fiber, a power transfer from one light wave to the other can be measured; since the acoustic propagation directly shifts with strain and temperature of the fiber, these two quantities can be measured by tuning the light waves' frequency offset.

\section{DISTRIBUTED BRILLOUIN SENSING: A SYSTEM OVERVIEW}

The most common technique to acquire the spatially resolved profile of Brillouin interaction along an optical fiber is the Brillouin optical time domain analysis (BOTDA): One of the light waves is injected in the form of very short pulses $(<10 \mathrm{~ns})$; by recording the scattering signal over time, the location of a scattering event can be reallocated from the time of flight of the optical pulses. Devices using this technique are commercially available.

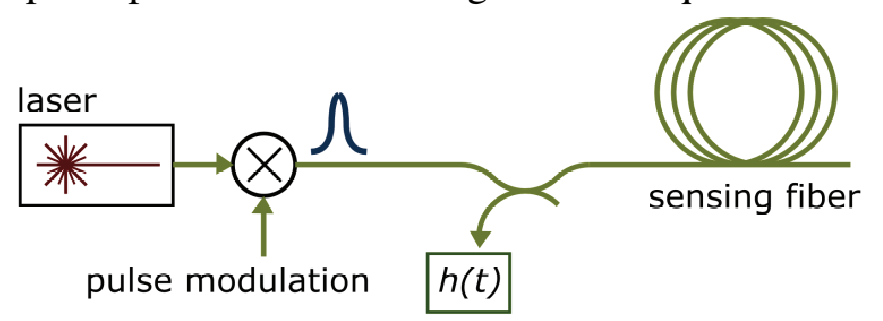

Figure 1: Distributed optical fiber sensing in the time domain, recording the fiber's pulse response $h(t)$

An alternative technique is the Brillouin optical frequency domain analysis (BOFDA). Here, the pulses are replaced by sinusoidal waves of a tunable frequency (ca. $5 \mathrm{kHz}$ to $100 \mathrm{MHz}$ ). By measuring the system's response to these frequencies (being the spectral components of the optical pulses in a harmonic representation), the transfer function of Brillouin interaction along the fiber is recorded. As in a linear, time-invariant system, the pulse response can be entirely constructed from the system's transfer function, a Fourier transform of the measured data will 
yield the same strain and temperature profile along the fiber that would have been acquired by the use of pulses.

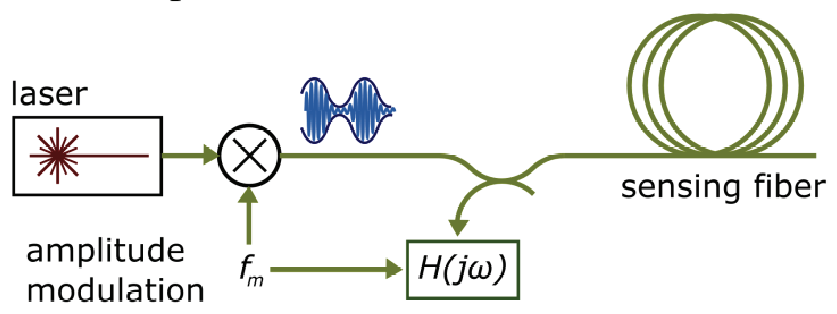

Figure 2: Distributed fiber sensing in the frequency domain by measuring the complex transfer function $\mathbf{H}(\mathrm{j} \omega)$

In both systems, the signal chain, going from the physical quantities to be measured to the final analysis giving insight into structural integrity, requires the following key elements:

- For deformation measurements in geotechnical or rigid structures, a reliable force transfer from the surrounding medium (soil, concrete, etc.) to the sensing fiber is needed. In most applications, this implies a rugged, but flexible coating material of the sensing cable, protecting the fiber from mechanical damage while still maintaining its sensitivity.

- Since Brillouin measurements are sensitive to both mechanical strain and temperature in the optical fiber, the temperature influence on deformation measurements must be accounted for. In contrast to strain-free buffered redundant fibers for temperature compensation within the sensing cable, the use of a distributed temperature measurement (DTS), based on Raman scattering, is free from cross-influences of mechanical friction on the temperature reference. Although this method lacks efficiency (two separate devices for distributed measurements are employed), it provides a reliable way to compensate a distributed Brillouin measurement for unwanted temperature effects and will be further discussed below.

- In the measurement unit itself, where the optical signals are generated and received, bears the task of converting the real-time occurrences of Brillouin interaction into a signal trace showing mechanical strain versus fiber length. In BOTDA devices, this is done by analog recording of the backscattered light for each pulse that has been injected into the fiber. In contrast, the BOFDA approach allows to sample each spectral component in the frequency domain, and then to continue all following signal processing steps offline. The implications of this advantageous long-way round are briefly described in the following.

\section{IMPLEMENTATION ISSUES OF THE BOFDA SYSTEM}

While the first publications on the BOFDA system had to rely on bulky, analog vector network analyzers, the measurements presented here were performed with a novel all-digital implementation of the BOFDA system. The key advantages in implementation when compared to state-of-the-art BOTDA systems are:

- By measuring the spectral components of the signal pulses one after another, frequency-domain filtering not only decreases noise within the received signal, but also unwanted characteristics of optical and electrical components which would not be distinguishable from the signal in the time domain. 
- The digital implementation allows for the use of state-of-the-art electronic devices such as fast signal converters and embedded signal processing. This makes the measurement unit small, more robust and efficient both in cost and power consumption.

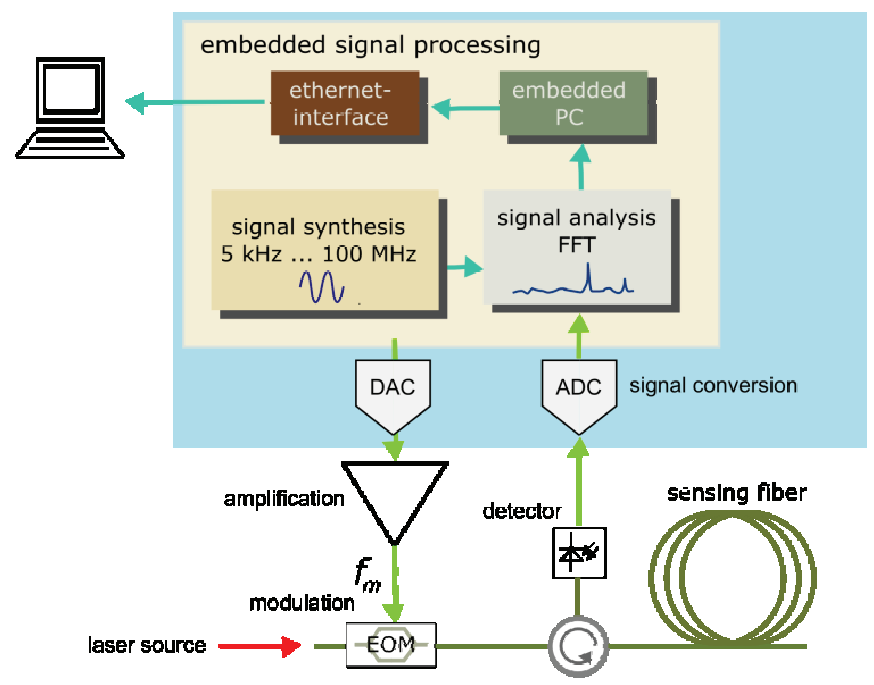

Figure 3: Setup of the all-digital implementation of the BOFDA system.

\section{DEFORMATION MEASUREMENTS FOR BOREHOLE MONITORING}

Distributed measurements have been performed in a borehole down to a depth of $800 \mathrm{~m}$. Two separate loops of single-mode fiber for Brillouin measurements and multimode fiber for Raman DTS (giving a total of four redundant traces for each technique), protected by a steel tube sensor cable, had been installed at the outside of the well casings. Measurements were performed using a state-of-the-art BOTDA device and the all-digital BOFDA setup for DTSS measurements and a Raman DTS device for temperature compensation. The BOTDA measurement was performed with a spatial resolution of $1 \mathrm{~m}$; the BOFDA measurement had a spatial resolution of $4.3 \mathrm{~m}$ and Raman DTS data were acquired with a spatial resolution of $1 \mathrm{~m}$.

Within a 5-day period, nine measurement traces were recorded with each device. Changes in the temperature distribution were externally induced by variation of fluid injection into the well. Figure 4 shows the results from two measurements with approx. 6 hours in between. The traces in the upper graphs correspond to the distribution of Brillouin interaction (BOFDA, BOTDA) and Raman interaction (DTS) along the fiber loop running down the well and back up again. A significant increase in temperature towards the bottom of the well is visible in all traces. With a temperature coefficient for the Brillouin shift (obtained from laboratory measurements using a temperature chamber) $\mathrm{df}_{\mathrm{B}} / \mathrm{dT}=0.9 \mathrm{MHz} /{ }^{\circ} \mathrm{C}$, the Brillouin DTSS measurements can be directly associated to the Raman DTS measurements. By subtracting the Raman DTS data, the compensated traces of Brillouin frequency shift in the lower figures are obtained. Apart from a slight drift in Brillouin shift in the BOTDA traces along the fiber length, the remaining fluctuations in Brillouin frequency lie within $1 \mathrm{MHz}$, which corresponds to a temperature uncertainty $<1{ }^{\circ} \mathrm{C}$. The compensated traces might now be interpreted in terms of mechanical stress along the well casing, provided that deformations are transferred to the optical fibers contained in the sensor cable. 

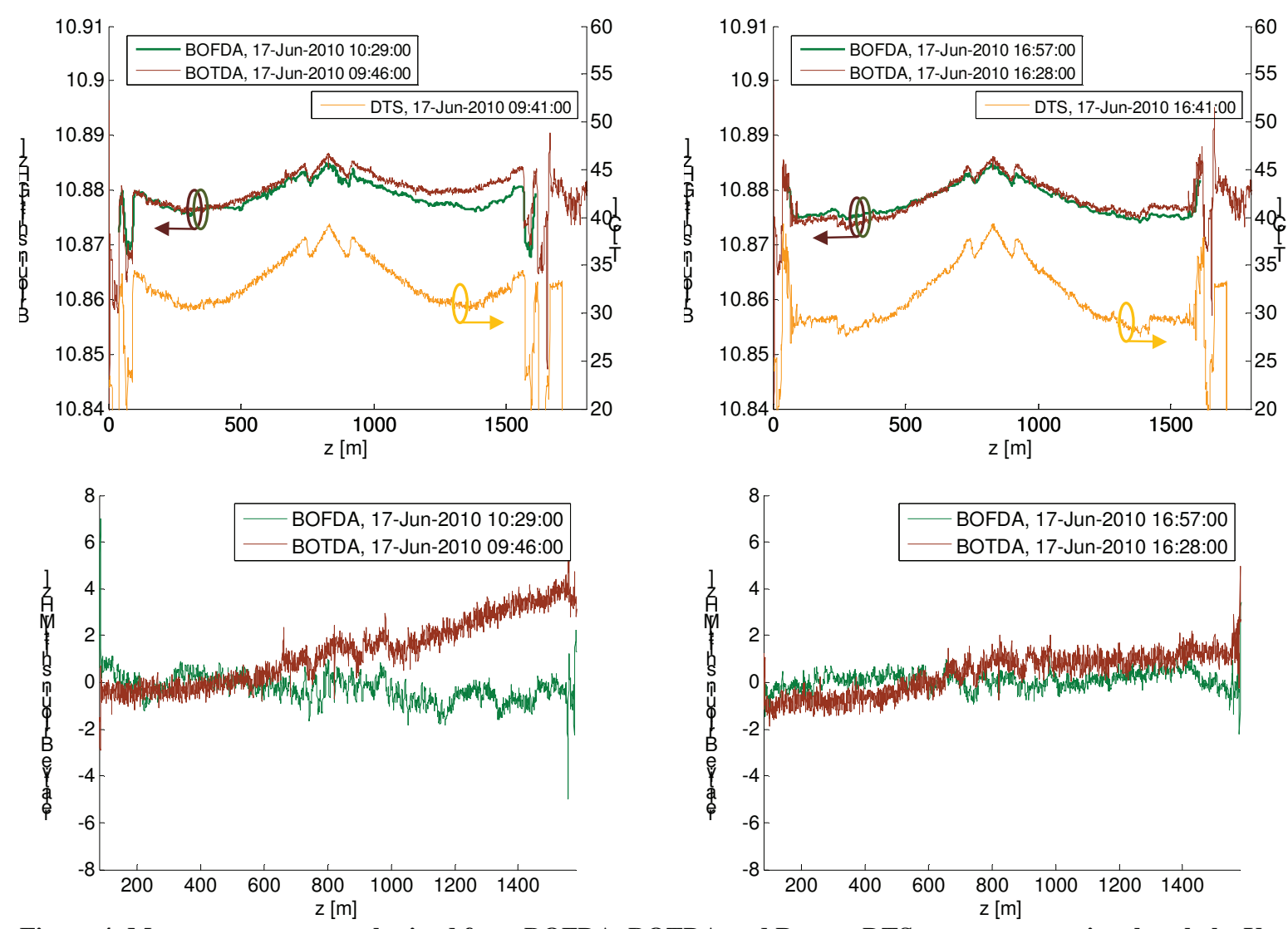

Figure 4: Measurement traces obtained from BOFDA, BOTDA and Raman DTS measurements in a borehole. Upper figures: Distribution of Brillouin and Raman interaction along the fibers (the arrows indicate the corresponding $y$ axis of each trace). Lower figures: BOFDA and BOTDA traces temperature-compensated by means of the Raman DTS measurement.

\section{CONCLUSIONS AND OUTLOOK}

The all-digital implementation of the Brillouin optical frequency domain analysis provides an efficient alternative to state-of-the-art Brillouin systems working in the time domain. Measurements along the well casings of a borehole have verified the applicability of both systems. Data interpretation with respect to geotechnical effects within the well will be subject of further research.

It has been shown that temperature compensation of a Brillouin measurement by means of a Raman DTS measurement can provide a reliable way to perform distributed measurements of mechanical deformation in optical fibers. However, in order to enhance efficiency of distributed strain measurements, further research on separation of temperature and strain within one single measurement unit is encouraged.

\section{LITERATURE}

1. N. Nöther, A. Wosniok, K. Krebber, and E. Thiele: "A distributed fiber-optic sensing system for monitoring of large geotechnical structures", ISHMII, 2009

2. Zhi Zhou, Jianping He, Minghua Huang, Jun He, and Genda Chen: "Casing Pipe Damage Detection with Optical Fiber Sensors: A Case Study in Oil Well Constructions, Advances in Civil Engineering", Volume 2010 